\title{
Enhancing the Anti-Tumor Effects of Cancer Peptide Vaccine Therapy
}

Norihiro Fujinami, Yu Sawada, Daisuke Nobuoka ${ }^{1}$ and Tetsuya Nakatsura*

Division of Cancer Immunotherapy, Exploratory Oncology Research \& Clinical Trial Center, National Cancer Center, Kashiwa, Chiba, Japan

*Corresponding author: Tetsuya Nakatsura, Division of Cancer Immunotherapy, Exploratory Oncology Research \& Clinical Trial Center, National Cancer Center, Kashiwa, Chiba, Japan, Tel: +81-4-7131-5490; Fax: +81-4-7133-6606; E-mail: tnakatsu@east.ncc.go.jp

Received date: Jul 13, 2016; Accepted date: Jul 25, 2016; Publication date: Jul 28, 2016

Copyright: (C) 2016 Fujinami N, et al. This is an open-access article distributed under the terms of the Creative Commons Attribution License, which permits unrestricted use, distribution, and reproduction in any medium, provided the original author and source are credited.

\begin{abstract}
The clinical efficacy of cancer peptide vaccines has been considered inadequate. To enhance the anti-tumor effects of peptide vaccines, we have studied effective enhancement methods for peptide vaccine therapies such as intratumoral peptide injection and combination therapies with anti-PD-1 blocking antibody or anti-CD4 depletion antibody. We aim to present effective clinical applications of peptide vaccines.
\end{abstract}

Keywords: Peptide vaccine; Glypican-3; Intratumoral peptide injection; Anti-PD-1 blocking antibody; Anti-CD4 depletion antibody

\section{Introduction}

Cancer peptide vaccine therapies can be used to prolong survival while maintaining the quality of life (QOL) in patients and are expected to prevent or reduce recurrences. We have previously reported that glypican-3 (GPC3) is a cancer specific antigen [1-3] Studies have identified GPC3-derived peptides that are capable of inducing peptide-specific cytotoxic $\mathrm{T}$ lymphocytes (CTLs) [4-6]. Several clinical trials for the GPC3 peptide vaccine therapy have been performed in hepatocellular carcinoma (HCC) [7-9]. Through previous studies, we have confirmed the safety and immunological efficacy of the vaccine and demonstrated its potential in inducing clinical effects in some patients [8-10]. However, the clinical efficacy of cancer peptide vaccine therapies is still considered inadequate. Consequently, we have attempted to develop effective enhancement methods for peptide vaccine therapies.

\section{Intratumoral peptide injection enhances tumor cell antigenicity}

Antigen-specific cancer immunotherapy involves antigen-specific CTLs, which recognize the antigen-derived peptides bound to major histocompatibility complex (MHC) class I molecules on the tumor cell surface and destroy the tumor cells. The low density of presented antigen bound to MHC class I molecules is one of the reasons why antigen-specific cancer immunotherapy has been ineffective in a clinical setting. We confirmed that most of the tumors exhibited enhanced expression of the human leukocyte antigen (HLA) class I molecules and its expression within the tumor area was higher than that outside it. Therefore, to induce additional peptide loading onto MHC class I molecules present on the tumor cells, we performed the intratumoral peptide injection for effectively enhancing the anti-tumor effect of peptide vaccines.

Intratumoral peptide injection was effective in inhibiting tumor growth and prolonging survival time. Furthermore, an antigenspreading effect was detected after the peptide injection, which enhances tumor cell antigenicity and may be a valuable option in improving the anti-tumor effects of antigen-specific cancer immunotherapy against solid tumors [11].

\section{Programmed death-1 (PD-1) blockade enhances the antitumor effects of peptide vaccines}

PD-1 is expressed on activated $\mathrm{T}$ and $\mathrm{B}$ cells, and it induces inhibitory signals [12]. Several studies have shown that the PD-1/PDL1 pathway plays a critical role in compromised tumor immunity $[13,14]$.

We have used peptide emulsified with incomplete Freund's adjuvant (IFA) for peptide vaccines in both animal models and clinical models. However, the peptide/IFA vaccination increased antigen-driven expression of the inhibitory receptors PD-1, LAG-3, CTLA-4, and Tim-3 in CTLs [15]. PD-1 blockade could partially rescue CTLs in a state of exhaustion. Therefore we employed the combination therapy by using the peptide vaccine and PD-1 blocking antibody. We demonstrated that PD-1/PD-L1 blockade enhanced the anti-tumor effects of peptide vaccines by increasing the immune response of vaccine-induced CTLs [16].

\section{Anti-tumor effects of peptide vaccines were enhanced in combination with anti-CD4 antibody}

Several studies have suggested that the depletion of CD4+ cells results in strong anti-tumor effects in tumor-bearing mice models due to the enhancement of CTL responses [17-19].

To enhance the anti-tumor effects of peptide vaccines, we included an anti-CD4 monoclonal antibody (mAb) (clone: GK1.5) in a mouse model. Using the IFN-- $\gamma$ ELISPOT assay, we determined that the number of ovalbumin (OVA)-specific CTLs inducted by OVA peptide vaccine in combination with anti-CD4 $\mathrm{mAb}$ was higher than that inducted by OVA peptide vaccine alone. Additionally, after the combined treatment with OVA peptide vaccine and anti-CD4 $\mathrm{mAb}$, perforin and granzyme secretion from CD107a+ cells increased and the production of IL-2 and TNF from these CTLs increased as analyzed by the CD107a assay and cytokine assay, respectively

Finally, we observed that metastasis was remarkably suppressed by the peptide vaccine in combination with anti-CD4 $\mathrm{mAb}$ in a murine model of liver metastasis. The mouse model of liver metastasis was 
developed by injecting tumor cells into the spleen. The evaluation of liver metastasis was performed using the weight of the murine liver, because the number of metastases in the liver could not be counted too many. The liver weight of the combination group treated with the OVA peptide vaccine and anti-CD4 $\mathrm{mAb}$ was significantly lower than those of the untreated group and the group treated with OVA peptide vaccine alone. However, the liver weight of the combination group showed no significant difference from that of the group treated with anti-CD4 mAb alone [20]. However, we believe that the enhanced inhibitory effects on metastasis in the combination therapy was derived from the enhancement in the multi-functionality of peptidespecific CTLs as determined by the IFN- $\gamma$ ELISPOT assay, CD107a upregulation assay and cytokine assay. Further investigations must be conducted to evaluate liver metastasis based on the number of liver metastases.

\section{Conclusion}

Although peptide vaccines exhibit disadvantages such as weak antitumor effects, they also have several advantages such as systemic effects similar to chemotherapies, with fewer side effects. Our therapeutic strategies such as intratumoral peptide injection or the combination therapies with antibody drugs can enhance the anti-tumor effect of cancer peptide vaccine therapy.

\section{Acknowledgments}

This study was supported in part by the National Cancer Center Research and Development Fund (25-A-7), and financial support from the Research on Applying Health Technology, the Research for Promotion of Cancer Control Programs, the Practical Research for Innovative Cancer Control from Japan Agency for Medical Research and Development (AMED 15ck0106002h0103, 15ck0106109h0002).

\section{References}

1. Nakatsura T, Yoshitake Y, Senju S, Monji, M, Komori H, et al. (2003) Glypican-3, overexpressed specifically in human hepatocellular carcinoma, is a novel tumor marker. Biochem Biophys Res Commun 306: 16-25.

2. Shirakawa H, Kuronuma T, Nishimura $\mathrm{Y}$, Hasebe T, Nakano M, et al. (2009) Glypican-3 is a useful diagnostic marker for a component of hepatocellular carcinoma in human liver cancer. Int J Oncol 34: 649-656.

3. Shirakawa H, Suzuki H, Shimomura M, Kojima M, Gotohda N, et al. (2009) Glypican-3 expression is correlated with poor prognosis in hepatocellular carcinoma. Cancer Sci 100: 1403-1407.

4. Komori H, Nakatsura T, Senju S, Yoshitake Y, Motomura Y, et al. (2006) Identification of HLA-A2- or HLA-A24-restricted CTL epitopes possibly useful for glypican-3-specific immunotherapy of hepatocellular carcinoma. Clin Cancer Res 12: 2689-2697.

5. Nakatsura T, Komori H, Kubo T, Yoshitake Y, Senju S, et al. (2004) Mouse homologue of a novel human oncofetal antigen, glypican-3, evokes T-cellmediated tumor rejection without autoimmune reactions in mice. Clin Cancer Res 10: 8630-8640.

6. Nakatsura T, Kageshita T, Ito S, Wakamatsu K, Monji M, et al. (2004) Identification of glypican-3 as a novel tumor marker for melanoma. Clin Cancer Res 10: 6612-6621.
7. Yoshikawa T, Nakatsugawa M, Suzuki S, Shirakawa H, Nobuoka D, et al. (2011) HLA-A2-restricted glypican-3 peptide-specific CTL clones induced by peptide vaccine show high avidity and antigen-specific killing activity against tumor cells. Cancer Sci 102: 918-925.

8. Sawada Y, Yoshikawa T, Nobuoka D, Shirakawa H, Kuronuma T, et al. (2012) Phase I trial of a glypican-3-derived peptide vaccine for advanced hepatocellular carcinoma: immunologic evidence and potential for improving overall survival. Clin Cancer Res 18: 3686-3696.

9. Sawada Y, Yoshikawa T, Fujii S, Mitsunaga S, Nobuoka D, et al. (2013) Remarkable tumor lysis in a hepatocellular carcinoma patient immediately following glypican-3-derived peptide vaccination: an autopsy case. Hum Vaccin Immunother 9: 1228-1233.

10. Suzuki S, Shibata K, Kikkawa F, Nakatsura T (2014) Significant clinical response of progressive recurrent ovarian clear cell carcinoma to glypican-3-derived peptide vaccine therapy: two case reports. Hum Vaccin Immunother 10: 338-343.

11. Nobuoka D, Yoshikawa T, Takahashi M, Iwama T, Horie K, et al. (2013) Intratumoral peptide injection enhances tumor cell antigenicity recognized by cytotoxic $\mathrm{T}$ lymphocytes: a potential option for improvement in antigen-specific cancer immunotherapy. Cancer Immunol Immunother 62: 639-652.

12. Agata Y, Kawasaki A, Nishimura H, Ishida Y, Tsubata T, et al. (1996) Expression of the PD-1 antigen on the surface of stimulated mouse $\mathrm{T}$ and B lymphocytes. Int Immunol 8: 765-772.

13. Iwai Y, Ishida M, Tanaka Y, Okazaki T, Honjo T, et al. (2002) Involvement of PD-L1 on tumor cells in the escape from host immune system and tumor immunotherapy by PD-L1 blockade. Proc Natl Acad Sci U S A 99: 12293-12297.

14. Iwai Y, Terawaki S, Honjo T (2005) PD-1 blockade inhibits hematogenous spread of poorly immunogenic tumor cells by enhanced recruitment of effector T cells. Int Immunol 17: 133-144.

15. Hailemichael Y, Dai Z, Jaffarzad N, Ye Y, Medina MA, et al. (2013) Persistent antigen at vaccination sites induces tumor-specific CD8(+) $\mathrm{T}$ cell sequestration, dysfunction and deletion. Nat Med 19: 465-472.

16. Sawada Y, Yoshikawa T, Shimomura M, Iwama T, Endo I, et al. (2015) Programmed death-1 blockade enhances the antitumor effects of peptide vaccine-induced peptide-specific cytotoxic T lymphocytes. Int J Oncol 46: 28-36.

17. den Boer AT, van Mierlo GJ, Fransen MF, Melief CJ, Offringa R, et al. (2005) CD4+ T cells are able to promote tumor growth through inhibition of tumor-specific CD8+ T-cell responses in tumor-bearing hosts. Cancer Res 65: 6984-6989.

18. Chamoto K, Wakita D, Ohkuri T, Uchinami Y, Matsushima K, et al. (2010) 3-Methylcholanthrene-induced transforming growth factor-betaproducing carcinomas, but not sarcomas, are refractory to regulatory $\mathrm{T}$ cell-depletion therapy. Cancer Sci 101: 855-861.

19. Ueha S, Yokochi S, Ishiwata Y, Ogiwara H, Chand K, et al. (2015) Robust Antitumor Effects of Combined Anti-CD4-Depleting Antibody and AntiPD-1/PD-L1 Immune Checkpoint Antibody Treatment in Mice. Cancer Immunol Res 3: 631-640.

20. Fujinami N, Yoshikawa T, Sawada Y, Shimomura M, Iwama T, et al. (2016) Enhancement of antitumor effect by peptide vaccine therapy in combination with anti-CD4 antibody: Study in a mouse model. Biochem \& Biophys rep 5: 482-491. 